\title{
Fourier optics analysis of grating sensors with tilt errors
}

\author{
Onur Ferhanoglu, ${ }^{1}$ M. Fatih Toy, ${ }^{2}$ and Hakan Urey ${ }^{1, *}$ \\ ${ }^{1}$ Department of Electrical Engineering, Koç University, Sariyer, Istanbul 34450, Turkey \\ ${ }^{2}$ Ecole Polytechnique Federale de Lausanne (EPFL), Advanced Photonics Laboratory, CH-1015 Lausanne, Switzerland \\ ${ }^{*}$ Corresponding author: hurey@ku.edu.tr
}

Received February 16, 2011; revised March 17, 2011; accepted March 18, 2011; posted May 11, 2011 (Doc. ID 142783); published June 8, 2011

\begin{abstract}
Dynamic diffraction gratings can be microfabricated with precision and offer extremely sensitive displacement measurements and light intensity modulation. The effect of pure translation of the moving part of the grating on diffracted order intensities is well known. This study focuses on the parameters that limit the intensity and the contrast of the interference. The effects of grating duty cycle, mirror reflectivities, sensor tilt and detector size are investigated using Fourier optics theory and Gaussian beam optics. Analytical findings reveal that fringe visibility becomes $<0.3$ when the optical path variation exceeds half the wavelength within the grating interferometer. The fringe visibility can be compensated by monitoring the interfering portion of the diffracted order light only through detector size reduction in the expense of optical power. Experiments were conducted with a grating interferometer that resulted in an eightfold increase in fringe visibility with reduced detector size, which is in agreement with theory. Findings show that diffraction grating readout principle is not limited to translating sensors but also can be used for sensors with tilt or other deflection modes. (c) 2011 Optical Society of America
\end{abstract}

OCIS codes: $\quad 050.0050,070.0070,230.4685$.

Dynamic diffraction gratings can be fabricated using microfabrication techniques with high precision and provide subnanometer interferometric displacement detection capability for microelectromechanical system (MEMS)-based microsensors and microsensor arrays. Such gratings have many applications, such as atomic force microscope [1], thermal imaging devices [2], grating light valve displays [3] , MEMS biomolecular sensors [4] ], and Fourier transform spectrometers [3,5], and in telecommunication applications [3].

One of the most important parameters that determines the sensitivity and the dynamic range of a grating-based sensor is the interference fringe visibility. Various sensor parameters have a high impact on the fringe visibility. In this Letter, the effects of reference reflector tilt, grating duty cycle, mirror reflectivities, and the monitor photodetector size are studied analytically using Fourier optics theory and Gaussian beam optics. The effect of tilt of the moving part on fringe visibility is verified with a grating interferometer. Tilt error could be caused by surface stress or the natural vibration modes of the sensor. This Letter presents practical results and formulas that can be used by those designing optical systems using dynamic gratings for any of the applications listed previously.

Figure 1 illustrates dynamic phase gratings where the reflector is a MEMS device and the bottom reflector is a fixed grating. Light that is reflected off the grating and the reflector forms an interference from which displacement of the sensor could be monitored with a photodetector. While the sensor in Fig. 1(a) performs a pure translator motion, the cantilever-type sensor in Fig. 1(b) performs both translational and rotational motion. Alternately, the grating can be placed on the moving part and the reflector can be stationary or moving, and stationary grating parts can be intertwined, which are essentially the same for the purpose of this analysis.

Throughout this Letter, the diffraction grating sensor is modeled as a thin phase grating and scalar diffraction theory using paraxial approximation (i.e., the grating period is assumed much larger than the wavelength of light) and constant scaling factors are ignored. Large deflection analysis of dynamic gratings, where the far field intensity cannot be calculated using thin phase grating assumption was explained in our prior work [6]. The phase profile $(\Phi(x))$ of the sensor in Fig. 1(b) is modeled using a linear phase profile assuming that the reflector is located on a small, linearly displaced portion at the tip of a cantilever and can be expressed as

$$
\begin{aligned}
\Phi(x)= & t(x) R_{1} \exp (j 4 \pi / \lambda(g+x \theta))[\operatorname{rect}(x /(\Lambda-f)) \\
& * \operatorname{comb}(x / \Lambda)]+t(x) R_{2}[\operatorname{rect}(x / f) \\
& * \operatorname{comb}((x-\Lambda / 2) / \Lambda)],
\end{aligned}
$$

where $\lambda$ is the wavelength of illuminating source, $R_{1}$ and $R_{2}$ are respectively the reflectivities of the reference reflector and grating fingers, and $t(x)$ is an aperture function including the laser beam profile. Comb function is an array of $\delta$ (dirac-delta) functions and rect is the rectangular function as defined in [7]. For $\theta=g / L=0$, Eq. (1) simplifies to the phase function of the translatory sensor shown in Fig. 1(a). Using the Fraunhofer approximation, the far field intensity profile can be calculated by taking the square of the Fourier transform of Eq. (1):
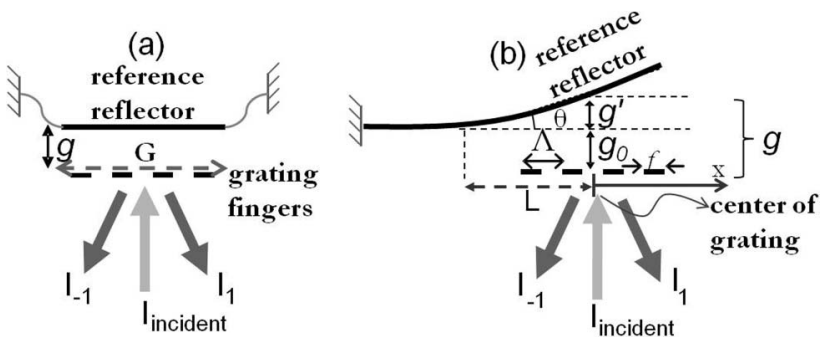

Fig. 1. Diffraction grating under a MEMS device. (a) Device having translational motion, (b) device having both translational and tilt motion. Incoming light and diffracted first-order lights are illustrated with arrows. Grating is along the $x$ axis, $\Lambda$ is the grating period, $f$ is the grating finger width, $G$ is the grating size, $g_{0}$ is the initial center gap, $g^{\prime}$ is the additional displacement due to tilt, $g=g_{0}+g^{\prime}$ is the total gap, and $\theta=g^{\prime} / L$ is the grating tilt angle, where $L$ is the distance from the middle of the grating to the virtual anchor point for the cantilever beam. 
$I(u)=\left|R_{1} \exp (j 4 \pi g / \lambda)[(1-D) \operatorname{sinc}((1-D) \Lambda u) \cdot \operatorname{comb}(\Lambda u)] * T(u-2 \theta / \lambda)+R_{2} \exp (j \pi u \Lambda)[D \operatorname{sinc}(D \Lambda u) \cdot \operatorname{comb}(\Lambda u)] * T(u)\right|^{2}$,

where $u$ is spatial frequency $\left(\mathrm{m}^{-1}\right), \operatorname{sinc}(x)=\sin (\pi x) / \pi x$, $D=f / \Lambda$ is the grating duty cycle, and $T(u)$ is the Fourier transform of $t(x)$. Equation (2) is a general expression and simplifies to the well-known diffraction efficiency formulas for zeroth-order and odd diffraction orders under the following assumptions: $\theta=0$ and the grating is wide and illuminated with uniform profile $(t(x)$ is infinitely wide, thus $T(u)=\delta(u), R_{1}=R_{2}=1$, and $D=0.5)$ :

$$
\begin{gathered}
I(u=0)=\cos ^{2}(2 \pi g / \lambda), \\
I(u=m / \Lambda)=4 / m^{2} \pi^{2} \sin ^{2}(2 \pi g / \lambda),
\end{gathered}
$$

where $m$ is any odd integer. Even diffraction orders for a square phase grating vanish, since they correspond to the zero crossings of the sinc term in Eq. (2).

Fringe visibility $(\gamma)$ can be defined as below using the maximum and the minimum intensities when $g$ is varied. For the zeroth order $(u=0)$, assuming no tilt $(\theta=0)$ and wide grating with uniform illumination (i.e., $T(u)=$ $\delta(u)), \gamma$ can be calculated by simplifying Eq. (2):

$$
\gamma=\frac{I_{\max }-I_{\min }}{I_{\max }+I_{\min }}=\frac{2 D(1-D)}{R_{1} / R_{2}(1-D)^{2}+R_{2} / R_{1} D^{2}} .
$$

For different $R_{1}$ and $R_{2}$, maximum fringe visibility, based on Eq. (5), is observed when

$$
R_{1}(1-D)=R_{2} D .
$$

When $R_{1}=R_{2}$, the maximum fringe visibility is obtained at $D=0.5$ as expected. Similar behavior is observed for higher diffracted orders as well.

The following paragraphs analyze the effect of tilt and detector size on the fringe visibility of the diffracted orders when the grating is illuminated with a Gaussian profile laser defined as

$$
t(x)=\exp \left(-x^{2} / W_{0}^{2}\right) \cdot \operatorname{rect}(x / G),
$$

where $W_{0}$ is the beam radius at the grating plane and the beam is clipped with grating size $G$. The radius of curvature of the laser beam wavefront is omitted for our case, however, it could be incorporated into Eq. (7) as an additional phase term.

The reflected beam from the tilted sensor follows a different path from the beam that reflects off the grating. Figure 2(a) illustrates zeroth, first, and -1st diffracted orders under tilted reflector condition, observed at distance $z$ away from the grating sensor. Figures $\underline{2(b)}$ and 2 (c) are close-up views of constructive and destructive interference cases at the zeroth diffracted order. Detector size is critical in determining the fringe visibility $(\gamma)$ at the output. A detector that captures both beams [area 1 in Figs. 2(b) and 2(c)] exhibits a low $\gamma$ in its output since it also captures light that does not interfere, which creates bias and reduces the fringe visibility. On the other hand, a detector that captures only the interference area at the intersection [area 2 in Figs. 2(b) and 2(c)], rejects most of the bias and gives high $(\gamma)$.

An analytical expression can be obtained for the encircled power around the zeroth diffracted order by superposing two Gaussian beams at the detector plane due to reflections from the top and bottom surfaces of the sensor. To obtain a simplified expression, perfect reflectors $\left(R_{1}=R_{2}=1\right), D=0.5$, incident Gaussian beam having radius $W(\boldsymbol{z})$ with no clipping such that $G \gg W(\boldsymbol{z})$ is assumed. Two Gaussians are separated by a distance of $2 \theta z$ at the detector plane due to tilt (as in Fig. 2), and the detector area is taken as $L_{d} \times L_{d}$. The encircled power is given by

$$
P_{0} \sim A+B \cos (4 \pi g / \lambda) \exp \left(-2(\theta \boldsymbol{z} / W(\boldsymbol{z}))^{2}\right),
$$

where

$$
A=\operatorname{erfc}\left[-\frac{\sqrt{2}}{W(z)}\left(\frac{L_{d}}{2}-\theta z\right)\right]-\operatorname{erfc}\left[\frac{\sqrt{2}}{W(z)}\left(\frac{L_{d}}{2}-\theta z\right)\right]
$$

$$
B=\operatorname{erfc}\left[-\frac{L_{d}}{\sqrt{2} W(z)}\right]-\operatorname{erfc}\left[\frac{L_{d}}{\sqrt{2} W(z)}\right] .
$$

In Eqs. (9) and (10), erfc is the complimentary error function. Based on Eq. ( $)$ ), $\gamma$ for the zeroth-order power at the detector can expressed assuming $g$ is varied:

$$
\gamma=(B / A) \exp \left(-2(\theta z / W(z))^{2}\right) .
$$

It can be shown that Eq. (11) is also the fringe visibility $(\gamma)$ expression for all odd diffraction orders. For a large detector, which captures all the energy of the two beams

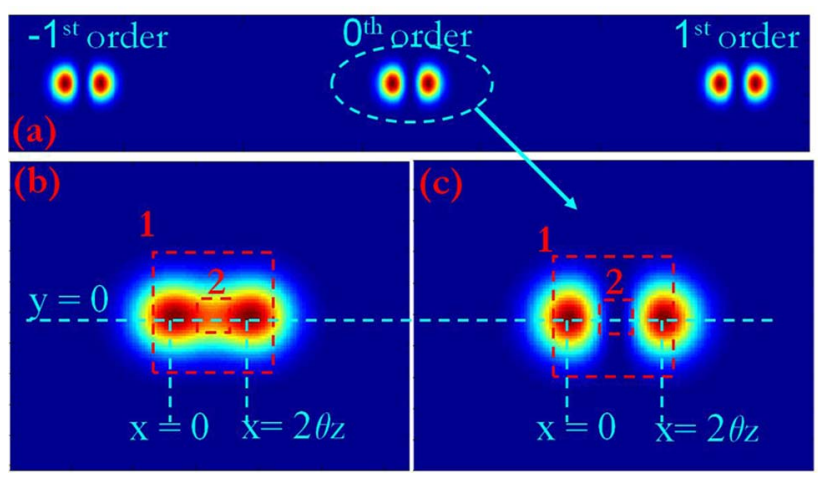

Fig. 2. (Color online) (a) Intensity profile for destructive interference when the sensor is tilted by an angle $\theta$ illustrating zeroth, first, and -1st diffracted orders. (b) Constructive interference at zeroth order. (c) Destructive interference at zeroth order. The detector that spans area 1 exhibits lower fringe visibility than the detector that spans area 2 . 


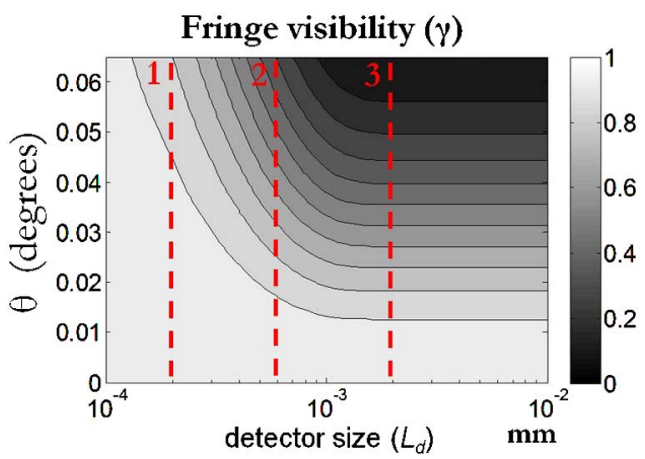

Fig. 3. (Color online) Fringe visibility contours of the detector output for different detector sizes and tilt angles. Lines 1 through 3 indicate detector sizes of $L_{d}=0.2,0.6$, $2 \mathrm{~mm}$ that are used in the experiments, where $\lambda=0.633 \mathrm{um}$, $W(z=0.4 \mathrm{~m})=400 \mathrm{um}$.

such that $L_{d}>2 \theta z$, without causing cross talk between diffraction orders, i.e., $L_{d}<\lambda z / \Lambda$, the error integrals $A$ and $B$ become equal. Equation (11) suggests a Gaussian decay of the visibility with tilt for all diffraction orders. Uniform illumination intensity instead of a Gaussian beam results in a sinc type of decay profile with tilt, as previously illustrated [8]. Based on Eq. (11), and wellknown Gaussian beam formulation [7], a fringe visibility $(\gamma)$ of $<0.3$ is observed at the far field, where $W(z) \approx$ $z \lambda / \pi W_{0}$ when the height difference within the interferometer is $W_{0} \theta=\lambda / 4$, which indicates a reversal in the phase of two extreme rays within the grating interferometer [9].

Figure 3 illustrates fringe visibility contours for different detector sizes and tilt angles. Experimental parameters: $\lambda=0.633 \mathrm{um}, W(z=0.4 \mathrm{~m})=400 \mathrm{um}$ is used while plotting Fig. 3. Higher $\gamma$ is observed when the detector size is within the intersection of two beams, where interference takes place (detector size indicated by line 1 in Fig. 3.). $\gamma$ decreases as the detector captures regions that are outside the intersection (line 3 in Fig. 3). Besides detector size, $\gamma$ also depends on the tilt of reference reflector. At small tilt angles, the effect of detector size on $\gamma$ becomes insignificant since the two beams fully coincide. As the tilt angle increases, the detector size needs to decrease in order to capture intersection area with high $\gamma$ at the cost of power.

Decrease in the fringe visibility is experimentally demonstrated with an amplitude grating and a back reflector. A translating mirror was placed at about $100 \mu \mathrm{m}$ from the grating of period $40 \mu \mathrm{m}$. This distance is much closer than the Talbot image distance of the grating, which is at about $2.5 \mathrm{~mm}$. The grating is on a precision tilt stage, and fringe visibility of the zeroth order was recorded with a large area photodetector placed $0.4 \mathrm{~m}$ away (sufficient distance since Gaussian beam profile is maintained everywhere) from the grating interferometer. Figure 4 illustrates the theoretical (shown as continuous functions) and the experimental results (shown as discrete points) of the change in fringe visibility with respect to tilt angle of the back reflector and photodetector sizes that are shown in Fig. 3. Experimental results match the theoretical model observed in Eq. (11) and similar decay

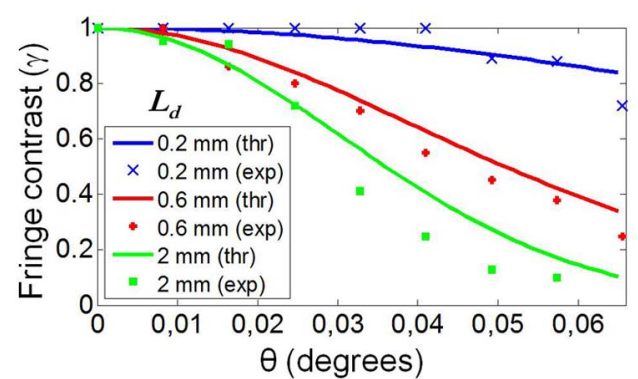

Fig. 4. (Color online) Zeroth-order fringe visibility $(\gamma)$ versus tilt angle of the back reflector and detector size for $\lambda=633 \mathrm{~nm}$, $W(z=0.4 \mathrm{~m})=400 \mathrm{um}$ for $L_{d}=0.2,0.6,2 \mathrm{~mm}$. Theoretical results are illustrated as continuous plots and experimental results are marked as discrete points.

behavior is observed for other orders as well. The discrepancies between the theory and the measurements are attributed to the error in manufacturing of the apertures using a printed circuit board prototyping machine and the handling precision of the manual tilt stage.

Study and experimental verification of diffraction order fringe visibility for grating-based tilted sensors with Gaussian illumination profile were presented. Simulations indicate that gratings with equal sensor and grating reflectivities and $50 \%$ duty cycle perform the best, as expected. It was observed that the fringe visibility decreases to $<0.3$ when the height difference due to tilt across the grating interferometer, i.e., $W_{0} \theta$ product, exceeds $\lambda / 4$. This study shows that diffraction grating readout principle is not limited to translating sensors but can also be used for sensors with tilt or other types of motion with some compromise in sensitivity. Decrease in fringe visibility may be corrected by using a small area detector at the expense of optical power. The change in the fringe visibility can also be used as a new method to detect small tilts in the sensor.

H. Urey acknowledges TUBA-GEBIP award. O. Ferhanoglu thanks TUBITAK for graduate fellowship.

\section{References}

1. A. G. Onaran, M. Balantekin, W. Lee, W. L. Hughes, B. A. Buchine, R. O. Guldiken, Z. Parlak, C. F. Quate, and F. L. Degertekin, Rev. Sci. Instrum. 77, 023501 (2006).

2. M. F. Toy, O. Ferhanoglu, H. Torun, and H. Urey, Sens. Actuators A Phys. 156, 88 (2009).

3. S. D. Senturia, D. R. Day, M. A. Butler, and M. C. Smith, J. Microlith. Microfab. Microsyst. 4, 041401 (2005).

4. H. Torun, K. K. Sarangapani, and F. L. Degertekin, IEEE J. Microelectromech. 19, 1021 (2010).

5. C. Ataman, H. Urey, and A. Wolter, J. Micromech. Microeng. 16, 2517 (2006).

6. O. Ferhanoglu, H. R. Seren, S. Lüttjohann, and H. Urey, Opt. Express 17, 21289 (2009).

7. J. W. Goodman, Introduction to Fourier Optics (Roberts, 2005).

8. T. Thundat, E. Finot, Z. Hu, R. H. Ritchie, G. Wu, and A. Majumdar, Appl. Phys. Lett. 77, 4061 (2000).

9. V. Saptari, Fourier Transform Spectroscopy Instrumentation Engineering (SPIE, 2003). 